\title{
Perimeter-Based Data Replication in Mobile Sensor Networks
}

\author{
Panayiotis Andreou*, Demetrios Zeinalipour-Yazti*, Maria Andreou ${ }^{\dagger}$, Panos K. Chrysanthis ${ }^{\ddagger}$, George Samaras* \\ *Department of Computer Science, University of Cyprus \\ P.O. Box 20537, 1678 Nicosia, Cyprus, \{panic, dzeina, cssamara\} @cs.ucy.ac.cy \\ ${ }^{\dagger}$ School of Pure and Applied Sciences, Open University of Cyprus \\ P.O. Box 24801, 1304 Nicosia, Cyprus, andreou@ouc.ac.cy \\ $\ddagger$ Department of Computer Science, University of Pittsburgh \\ Pittsburgh, PA 15260, USA, panos@cs.pitt.edu
}

\begin{abstract}
This paper ${ }^{1}$ assumes a set of $n$ mobile sensors that move in the Euclidean plane as a swarm. Our objectives are to explore a given geographic region by detecting spatio-temporal events of interest and to store these events in the network until the user requests them. Such a setting finds applications in mobile environments where the user (i.e., the sink) is infrequently within communication range from the field deployment. Our framework, coined SenseSwarm, dynamically partitions the sensing devices into perimeter and core nodes. Data acquisition is scheduled at the perimeter, in order to minimize energy consumption, while storage and replication takes place at the core nodes which are physically and logically shielded to threats and obstacles. To efficiently identify the nodes laying on the perimeter of the swarm we devise the Perimeter Algorithm (PA), an efficient distributed algorithm with a low communication complexity. For storage and fault-tolerance we devise the Data Replication Algorithm (DRA), a voting-based replication scheme that enables the exact retrieval of events from the network in cases of failures. Our trace-driven experimentation shows that our framework can offer significant energy reductions while maintaining high data availability rates. In particular, we found that when failures are less than $60 \%$ failure then we can recover over $80 \%$ of generated events exactly.
\end{abstract}

\section{INTRODUCTION}

Stationary sensor networks have been predominantly used in applications ranging from environmental monitoring [25], [23] to seismic and structural monitoring [5] as well as industry manufacturing [19]. Recent advances in distributed robotics and low power embedded systems have enabled a new class of Mobile Sensor Networks (MSNs) [6], [29] that can be used in land [2], [7], [16], ocean [17] and air [9] exploration and monitoring, automobile applications [11], [8], habitant monitoring [23] and a wide range of other scenarios. MSNs have a similar architecture to their stationary counterparts, thus are governed by the same energy and processing limitations, but are supplemented with implicit or explicit mechanisms that enable these devices to move in space (e.g., motor or sea/air current). Additionally, MSN devices might derive their coordinates through absolute (e.g., dedicated Geographic

\footnotetext{
${ }^{1}$ This work appears in the Proceedings of the 10th International Conference on Mobile Data Management Systems, Services and Middleware (MDM 2009).
}

Positioning System hardware) or relative means (e.g., localization techniques, which enable sensing devices to derive their coordinates using the signal strength, time difference of arrival or angle of arrival). The absence of a stationary network structure in MSNs makes continuous data acquisition to some sink point a non-intuitive task as data acquisition needs to be succeeded by in-network storage [30], [24], [1], such that these events can later be retrieved by the user. Additionally, the operation of MSNs is severely hampered by the fact that failures are omnipresent, thus fault-tolerance schemes become of prime importance in such environments.

In this paper we propose SenseSwarm ${ }^{2}$, a novel framework for the acquisition and storage of spatio-temporal events in MSNs. In SenseSwarm, nodes have the dual role of perimeter and core nodes (see Figure 1). Data acquisition is scheduled at the perimeter, in order to minimize energy consumption, while storage and replication takes place at the core nodes. Such a setting is suited well for applications in which new events are more prevalent at the periphery of the swarm (e.g., water and contamination detection) rather than for online monitoring applications (e.g., fire detection) or applications where new events might occur anywhere in the network. In our setting, storage of detected events takes place at the core nodes since these nodes are expected to feature a longer lifetime (due to their reduced sensing activity) but are also physically shielded to threats and obstacles that might immobilize the sensors. In order to increase the overall fault-tolerance of our system, we propose a data replication scheme that increases the availability of data and thus also the accuracy of executed queries.

For ease of exposition, let us now consider a Mars Exploration scenario: Spirit was one of the two rovers deployed by NASA in 2004 in order to perform geological analysis of the red planet. Instead of one rover, consider a design that consists of many cheaper rovers deployed as a swarm. Such a design avoids the peculiarities of individual rovers,

\footnotetext{
${ }^{2}$ The term Swarm (or Flock) in this paper refers to a group of objects that exhibit a polarized, non-colliding and aggregate motion.
} 


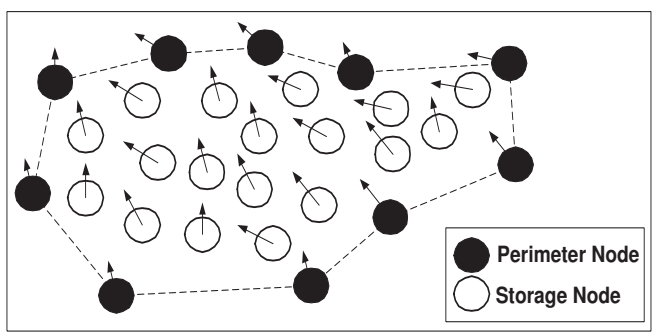

Fig. 1. SenseSwarm: Data Acquisition takes place at the virtual perimeter while core nodes act as storage nodes for the acquired events.

is less prone to failures and is potentially much cheaper. The swarm moves together and attempts to detect events of interest (e.g., the presence of water). Let the polarized behavior of the swarm be provided by an explicit algorithm [22] or an implicit mechanism (e.g., air current). The operator (on earth) then infrequently posts the question: "Has the swarm identified any water and where exactly?" Since the sink is located far away from the field deployment, the swarm collects spatio-temporal events of interest and stores them in the network until the operator requests them. In order to increase the availability of detected answers, in the presence of unpredictable failures, nodes perform data replication to neighboring nodes.

Similarly to the above visionary description, we could draw another more realistic example in the context of an ocean monitoring environment: assuming $n$ independent surface drifters floating on the sea surface and equipped with either acoustic or radio communication capabilities, the operator infrequently seeks to answer the query: "Has the swarm identified an area of contamination and where exactly?" Finally, one could utilize a swarm of car robots, such as CotsBots [2], Robomotes [7] or Millibots [16], to construct spatio-temporal acquisition and storage scenarios for land applications.

This paper builts upon our previous work in [28], in which we presented the initial design of the SenseSwarm framework. In this paper we introduce several new improvements including a voting-based fault-tolerance scheme that increases the availability of data and thus improves fault tolerance. In particular, our work makes the following contributions:

- We present the Perimeter Algorithm (PA), which efficiently constructs a perimeter of a MSN using a twophase protocol. Our algorithm has a $O(n)$ message complexity, where $n$ is the total number of sensors instead of $O\left(n^{2}\right)$, featured by the centralized algorithm.

- We devise a voting-based replication scheme to preserve the datums (i.e., acquired events) in cases of system failures. In particular, we devise the $D R A$ algorithm that replicates datums using distributed read/write quorums.

- We experimentally validate the efficiency of our propositions using a trace-driven experimental study that utilizes real sensor readings.

The remainder of the paper is organized as follows: Section II overviews the related research work and provides background on our perimeter construction and fault-tolerance scheme we present. Section III formalizes our system model and assumptions, Section IV the PA algorithm and Section V the DRA algorithm. Section VI presents our experimental study and Section VII concludes the paper.

\section{RELATED WORK AND BACKGROUND}

This section provides an overview of predominant data acquisition frameworks in order to highlight the unique characteristics of the SenseSwarm framework. It also provides background on the two main problems our framework addresses (i.e., the perimeter construction and the data replication processes).

Traditional data acquisition frameworks for sensor networks, such as TinyDB [18] and Cougar [26], perform a combination of in-network aggregation and filtering in order to reduce the energy consumption while conveying data to the sink. The MINT View framework [27] performs in-network top-k pruning in order to further reduce the consumption of energy. In data centric routing, such as directed diffusion [12], low-latency paths are established between the sink and the sensors. Contrary to our approach, all the above frameworks have been proposed for stationary sensor networks while this work considers the challenges of a mobile sensor network setting. In data centric storage schemes [24], [1], data with the same attribute (e.g., humidity readings) is stored at the same node in the network offering therefore efficient location and retrieval. Such an approach is supplementary to the perimeterbased data acquisition framework we propose in this paper. Supplementary to our framework are also the MicroHash [30] and TINX [20] local index structures, which provide $O(1)$ access to data stored on the local flash media of a sensor device. Such structures can be deployed to speed up the retrieval of data whenever required.

The first problem our framework investigates is that of partitioning the network into perimeter and core nodes. The perimeter construction problem we consider has similarities to the convex hull problem in computational geometry, which finds applications in pattern recognition, image processing and GIS [4]. The convex hull problem is defined as follows: given a set of points, identify the boundary of the smallest convex region that encloses all the points either on the boundary or on its interior. Such a boundary is both non-intersecting (i.e., no edge crosses any other edge) and convex (i.e., all internal angles are less than $\pi$ ). There are numerous centralized algorithms for computing the convex hull with varying complexities.

Two of the most popular convex hull algorithms are the Jarvis March [4] (or Gift Wrapping) algorithm and the Graham's scan algorithm [4]. The main difference between the convex hull and the perimeter problem we consider in this work, is that the latter defines non-convex cases (i.e., internal angles are up to $2 \pi$ ). Non-convex cases are typical for a sensor network context as convex angles might not be feasible due to communication radius constraints. Additionally, convex hull algorithms are centralized while we develop techniques to compute the boundaries in a distributed fashion minimizing communication and energy consumption without sacrificing 


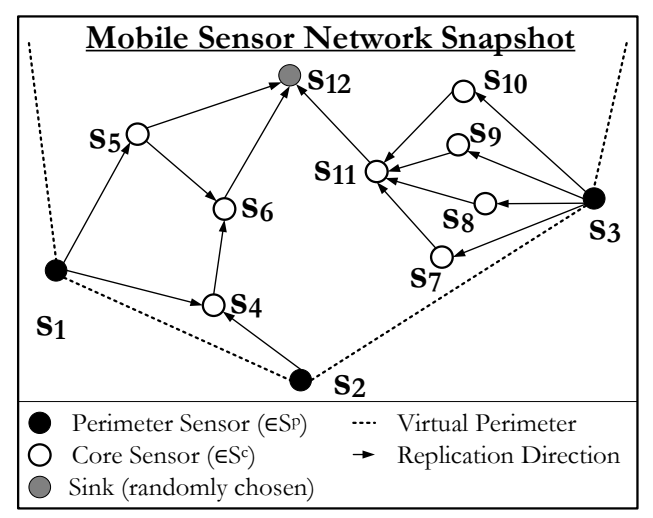

Fig. 2. In SenseSwarm, data acquisition is scheduled at the perimeter while storage and replication takes place at the core nodes.

correctness. Related work in the context of sensor networks appears in [3], where the authors present localized techniques that enable the sensors to determine whether they belong to the boundary of some phenomenon. Yet, the underlying assumption in the given work is that the edge sensors are not within communication range while we consider the perimeter to be a continuous chain of nodes. In [21], the authors devise an algorithm that combines current and historic measurements to trace a contour of a given value in the field (e.g., an oil spill). The presented ideas (e.g., that of quickly arriving at the contour) are supplementary to ideas presented in this paper.

The second problem our framework investigates is that of data replication to improve fault-tolerance. At a high level, our proposed scheme consists of maintaining a set of identical copies of each datum at several nodes in the network. For ease of exposition, let us consider the example network of Figure 2, which will be utilized throughout this paper. On the given figure we illustrate a segment of a MSN at a specific time $\tau$. Assume that a copy of the datum $d_{1}$ (i.e., data published by node $s_{1}$ ), has been replicated to nodes $s_{4}, s_{5}, s_{6}, s_{12}$. Now let nodes $s_{1}$ permanently fail along with its one hop neighbors (i.e., $s_{4}$ and $s_{5}$ ) at time instance $\tau+1$. Since $d_{1}$ has been replicated beyond these nodes then it will be feasible to recover $d_{1}$ if necessary.

Our proposed solution is based on a voting-based data replication scheme. Voting algorithms [14], [15] have been among the most popular techniques to offer fault-tolerant properties in distributed systems. A vote denotes the preference of some node to replicate a specific piece of information (i.e., a datum) to another node. Voting schemes consist of first selecting a set of nodes where a specific datum will be replicated (i.e., the write quorum) and another set of nodes where a query will be conducted at, to search for that specific datum (i.e., the read quorum). One of the major challenges is to effectively choose the correct quorums so that the replication process will produce consistent results in an efficient manner. SenseSwarm's data replication algorithm utilizes the basic ideas of voting in conjunction with the unique characteristics of MSN systems.
TABLE I

Definition of Symbols

\begin{tabular}{|c|l|}
\hline Symbol & Definition \\
\hline \hline$n$ & Number of Sensors $S=\left\{s_{1}, s_{2}, \ldots, s_{n}\right\}$ \\
$m$ & Number of attributes at each $s_{i}\left\{a_{1}, a_{2}, \ldots, a_{m}\right\}$ \\
$\left(s_{i}^{x}, s_{i}^{y}\right)$ & x and y coordinates of each $s_{i}$ \\
$r$ & The communication radius of each $s_{i}$ \\
$N H\left(s_{i}\right)$ & 1-hop (in commun. range) neighbors of $s_{i}$ \\
$V\left(s_{i}, s_{j}\right)$ & A Vector defined as $\left(s_{j}^{x}-s_{i}^{x}, s_{j}^{y}-s_{i}^{y}\right)$ \\
$\operatorname{LeftN}\left(s_{i}\right)$ & The predecessor of $s_{i}$ on the perimeter \\
$\left.\operatorname{RightN} \cos _{i}\right)$ & The successor of $s_{i}$ on the perimeter \\
$S^{p}, S^{c}$ & The set of Perimeter nodes, Core nodes \\
$Q$ & An m-dimensional Query \\
$e$ & Epoch Duration (i.e., data acquisition interval) \\
$\sigma, \sigma^{\prime}$ & Perimeter Reconstruction, Replication interval \\
$d_{i}$ & The datum of node $s_{i}$ \\
$v_{i}^{j}, v_{i}$ & The vote (preference) of $s_{i}$ to replicate $d_{i}$ \\
& to node $s_{j}$, All votes from $s_{i}$ \\
\hline
\end{tabular}

\section{System Model AND Assumptions}

In this section we will formalize our basic terminology and assumptions. The main symbols and their respective definitions are summarized in Table I.

Let $\Re \times \Re$ denote a two-dimensional grid of points in the Euclidean plane that discretizes a given geographic area. Also assume a Cartesian coordinate system to describe the position of each point in the grid with coordinates $(x, y)$. W.l.o.g, let us initialize the $n$ sensing devices $S=\left\{s_{1}, s_{2}, \ldots, s_{n}\right\}$ at the lower-left $n^{\frac{1}{2}} \times n^{\frac{1}{2}}$ sub-grid of $\Re^{2}$. For ease of exposition let $n$ be a perfect square such that each cell contains exactly one sensor. Each $s_{i}(i \leq n)$ can derive its coordinates $\left(s_{i}^{x}, s_{i}^{y}\right)$ through some absolute or relative mechanism. Additionally, each $s_{i}$ can be aware of its neighboring nodes, denoted as $N H\left(s_{i}\right)$, using a local 1-hop broadcast. The sensing devices are coarsely synchronized through some operating system mechanism (e.g., similarly to TinyOS [10]) or through the GPS and can communicate with other sensors in a uniform radius $r$, i.e., $1 \leq r \ll n^{\frac{1}{2}}$.

The user can specify one or more m-dimensional Boolean queries of the type $Q=\left\{q_{1} \odot q_{2} \odot \ldots \odot q_{m}\right\}$, where $q_{i}(i \leq m)$ corresponds to some predicate such as $q_{1}=$ "Temperature $>$ $100^{\prime \prime}$ and $\odot$ denotes some binary Boolean operator. These queries correspond to the user-defined local events of interest and are registered at each $s_{i}$ either prior the deployment or during execution. The discussion of more complex query types is outside the scope of this paper.

A SenseSwarm network is initiated by conceptually dividing $S$ into perimeter nodes $S^{p}$ and core nodes $S^{c}$ using the algorithms we present in Section IV. This operation is periodic and will be repeated after $\sigma$ time instances (see Figure 3). Each perimeter sensor $s_{i}(i \leq n)$ then acquires $m$ physical parameters $A=\left\{a_{1}, a_{2}, \ldots, a_{m}\right\}$ from its environment during every epoch $e$, which defines the interval after which data acquisition re-occurs. The value for $e$ is either dynamically adjusted according to the dynamics of the swarm or prespecified. In a sea oil-spill detection scenario, $e$ can be configured to several hours as surface drifters usually float very slowly 


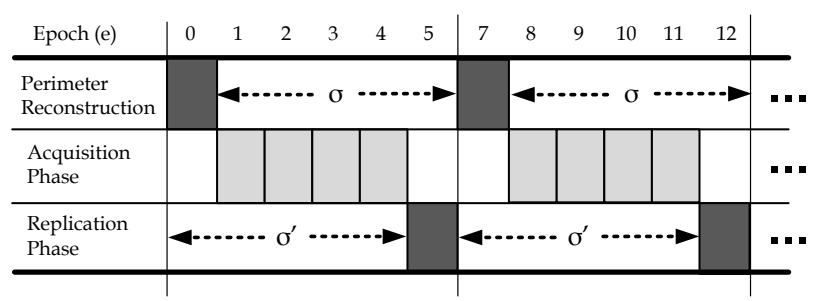

Fig. 3. Outline of the SenseSwarm framework operation.

on the sea surface. The above procedure generates spatiotemporal tuples of the form $\left\{t, x, y, a_{1}, a_{2}, \ldots, a_{m}\right\}$ locally at each sensor. The generated tuples of interest (with respect to $Q$ ) are stored in some local vector structure that will be referred to as $d_{i}$ (i.e., the datum of node $s_{i}$ ).

In order to increase the availability of $d_{i}$ structures, we adopt a data replication scheme based on votes that will be presented in Section V. A vote $v_{i}^{j}$ denotes the preference of sensor $s_{i}$ (i.e., the publisher of some datum $d_{i}$ ), to replicate $d_{i}$ to node $s_{j}(i \neq j)$ at a given time instance. Additionally, we define $v_{i}$ as the set of all votes by node $s_{i}$ on the given time instance.

In our approach, we assume that every $\sigma^{\prime}$ time instances every sensor $s_{i} \in S^{p}$ proceeds with the replication of its local datum $d_{i}$ to the votes of $s_{i}$.

\section{Perimeter Construction Phase}

This section describes algorithms for the construction of a perimeter in a MSN. We first describe a centralized solution and then our Perimeter Algorithm.

\section{A. Centralized Perimeter Algorithm (CPA)}

First note that the construction and dissemination of a perimeter can be performed in a centralized manner, i.e., a sink collects the coordinates of all nodes in $S$, using an adhoc spanning tree, and then identifies the perimeter nodes $\left(S^{p}\right)$ using some straightforward geometric calculations. Finally, the sink disseminates the ordered set $S^{p}$ to all nodes in $S$ using a spanning tree. Clearly, the first and last phase of the CPA algorithm require the transfer of many $(x, y)$-pairs between nodes. Specifically, although both phases require $\mathrm{O}(\mathrm{n})$ messages the first phase requires the transfer of $\mathrm{O}\left(n^{2}\right)(x, y)$ pairs (i.e., assume that the nodes are connected in a bus topology which yields $\sum_{1}^{n}(i)=\frac{n(n+1)}{2}(x, y)$ pairs $)$, while the last phase requires the transfer of $\mathrm{O}(p * n)(x, y)$-pairs (i.e., each edge transfers the complete perimeter of size $p$ ).

\section{B. Perimeter Algorithm (PA)}

We shall next describe our distributed algorithm which minimizes the transfer of $(x, y)$-pairs, thus minimizing energy consumption. To simplify the description and w.l.o.g., assume that we have no coincidents (i.e., two points with the same $(x, y)$ coordinates) and that no three points are collinear (i.e., lie on the same line). Although these assumptions make the discussion easier our implementation supports them.

Algorithm 1 presents the steps of the distributed PA process that is executed by each sensor every $\sigma$ time instances. In

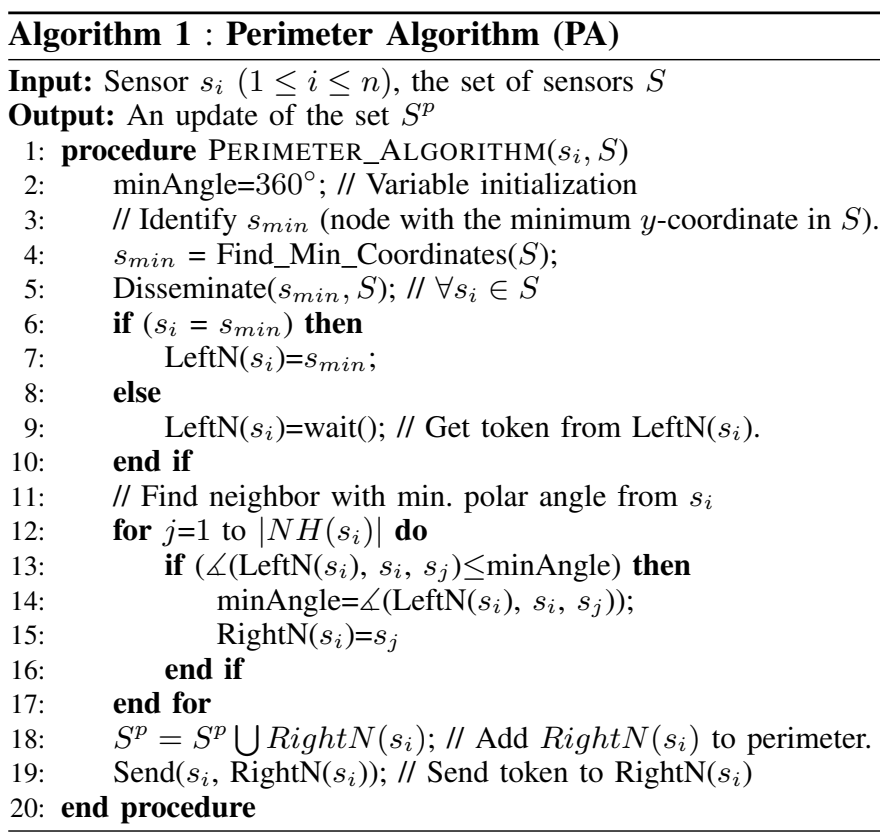

line 4, procedure Find_Min_Coordinares $(S)$ identifies the sensor with the minimum $y$-coordinate and returns its $i d$ to the variable $s_{\min }$. If more than one sensors have the $y$-coordinate equal to $s_{m i n}^{y}$, then the above procedure returns the one with the minimum value in its $x$-coordinate. The above procedure is achieved by constructing an aggregation tree rooted at the given sink using TAG [19]. In particular, each $s_{i}$ identifies among its children and itself the minimum $s_{\min }^{y}$ value and then recursively forwards the triple $\left(s_{\min }, s_{\min }^{x}, s_{\min }^{y}\right)$ to $s_{i}$ 's parent. This step, has similarly to CPA, a message complexity of $O(n)$ but the overall number of $(x, y)$-pairs transmitted to the sink is only $O(n)$ rather than $O\left(n^{2}\right)$ (i.e., exactly one pair per edge). This improvement is due to the in-network aggregation that takes place in our approach.

Concurrently with the above operation in line 4 , each $s_{i}$ updates its neighbor list $N H\left(s_{i}\right)$ as such an updated list will be necessary in the subsequent steps. Note that this update does not introduce any extra cost, as $s_{i}$ simply adds to $N H\left(s_{i}\right)$ the neighbors that have participated in the calculation of $s_{\text {min }}$.

In line 5 , we disseminate $s_{\min }$ to all the nodes in the network $S$ from the sink. This has a message complexity of $O(n)$ and the overall number of $(x, y)$-pairs transmitted is $O(n)$, compared to $O(p * n)$ required by CPA. The next task is to identify the nodes on the perimeter. Before proceeding, let us provide the following definitions:

Definition 1 [Left Neighbor of $\left.s_{i}\left(\operatorname{LeftN}\left(s_{i}\right)\right)\right]$ : The predecessor of $s_{i}$ on the perimeter. The termination condition of this recursive definition is as follows: $\operatorname{LeftN}\left(s_{\min }\right)=s_{\text {min }}$, where $s_{\text {min }}^{y} \leq s_{j}^{y}\left(\forall s_{j} \in S, 1 \leq j \leq n\right)$.

Definition 2 [Right Neighbor of $s_{i}\left(\operatorname{Right} N\left(s_{i}\right)\right)$ ]: The successor of $s_{i}$ on the perimeter such that $\operatorname{LeftN}\left(s_{i}\right) \neq$ $\operatorname{RightN}\left(s_{i}\right)$, if $\left|N H\left(s_{i}\right)\right|>1$.

Continuing with the description of our algorithm in lines 


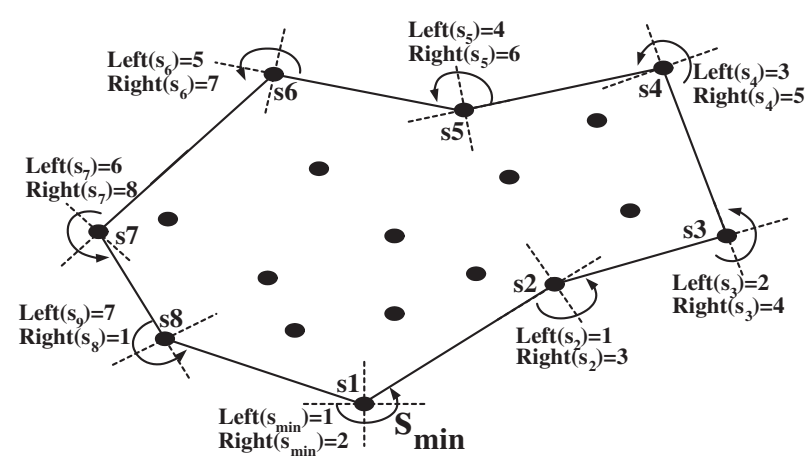

Fig. 4. Execution of PA: The construction starts at $s_{\min }$ and proceeds counterclockwise starting from $\pi$.

8-10 each $s_{i}$, other than $s_{\text {min }}$, identifies its left neighbor. This is achieved by waiting for a token (i.e., the identifier of $\operatorname{LeftN}\left(s_{i}\right)$ ) from $\operatorname{LeftN}\left(s_{i}\right)$. When the token arrives, the node will execute the remaining steps of the algorithm (lines 12-19). In particular, in lines $12-17, s_{i}$ identifies the neighbors with the minimum polar angle from its $x$-axis. The $x$-axis of node $s_{i}$ is defined in our context to be collinear with the vector $V\left(\operatorname{LeftN}\left(s_{i}\right), s_{i}\right)$. This ensures the correctness of the algorithm although we omit a formal proof due to space limitations. In line 15 we utilize the notation $\measuredangle(a, b, c)$ to denote the angle between three arbitrary points $a, b, c$ in the plane. Our objective in the given block (line 13-18), is to identify the neighbor with the minimum polar angle (which is then coined $\operatorname{RightN}\left(s_{i}\right)$ ), counterclockwise starting from $\pi$. Finally in line $19, s_{i}$ transmits a token to $\operatorname{RightN}\left(s_{i}\right)$ notifying it that it is the next node on the perimeter. The procedure between lines 12-20 continues sequentially along the network perimeter until any $s_{i}$ receives the token for a second time from its left neighbor or a timeout period expires. At the end, every node receiving the token knows that it belongs to $S^{p}$ while the rest nodes continue to belong to $S^{c}$.

The identification of $s_{\text {min }}$ takes $O(n)$ messages and the token dissemination takes $O(p)$ messages, where $p$ the number of the nodes on the perimeter. Thus the overall message complexity is $O(n)$, as $p \leq n$. In the future we plan to devise techniques to incrementally compute the perimeter.

Example: Figure 4, illustrates the perimeter construction for eight nodes $\left\{s_{1} \cdots s_{8}\right\}$. Assume that we have executed steps 2-5 of Algorithm 1 and that we continue with the execution of the perimeter construction at node $s_{\min }$ (i.e., $s_{1}$ ). $s_{\min }$ measures the polar angle of all the nodes in $N H\left(s_{\text {min }}\right)$ to its $x$-axis and subsequently derives $\operatorname{Right} N\left(s_{\min }\right)=2\left(s_{3}\right.$ is not within communication range from $s_{1}$ ). Next, $s_{\text {min }}$ sends a token to $s_{2}$ informing it that it is the next node on the perimeter. Upon reception of the token, $s_{2}$ sets its $x$-axis collinear with $V\left(s_{1}, s_{2}\right)$. The same idea applies to all nodes on the perimeter until $s_{8}$ transmits the token to $s_{1}$.

\section{ACQuisition ANd Data Replication Phase}

In this section we describe the second phase of the SenseSwarm Framework during which the perimeter nodes $S^{p}$ start acquiring information from their environment and then replicate this information to their neighboring nodes.

Recall that the acquisition step proceeds every $e$ time instances during which each $s_{i}$ generates spatio-temporal tuples of the form $\left\{t, x, y, a_{1}, a_{2}, \ldots, a_{m}\right\}$. The generated tuples of interest (i.e., the tuples that satisfy the predicates of $Q$ ) are recorded in the local $d_{i}$ (datum) structure of each $s_{i}$. Next, $d_{i}$ structures are replicated to neighboring nodes according to the algorithm we propose in this section. In particular, we propose a data replication scheme based on votes.

The presented DRA algorithm replicates the $d_{i}$ structures to $w$ neighboring nodes (for any $w \geq 1$ ). If it is necessary to recover $d_{i}$ then it is required to read $d_{i}$ structures from at least $r=v-w+1$ votes of $s_{i}$, where $v$ is the total number of votes of $s_{i}$. For instance when $w=2$ and $v=4$ then $r=4-2+1=3$ (i.e., 3 reads) are adequate to recover $d_{i}$ in its exact form. When $w=1$ and $v=4$ then $r=4-1+1=4$ reads are necessary to recover any replicated $d_{i}$. The details of the DRA algorithm follow next.

\section{A. Data Replication Algorithm (DRA)}

The objective of the DRA algorithm is to construct a data replication configuration which will present to each $s_{i}$ an energy efficient plan on how to replicate its local $d_{i}$ structures. A data replication configuration is an energy efficient (read,write)-combination that dictates how many read and writes operations are necessary per $d_{i}$, such that a $d_{i}$ structure can be preserved in cases of failures. It is important to notice that if energy conservation was not important then we could have opted for a scheme that replicates each $d_{i}$ to the entire network.

Algorithm 2 presents the details of the DRA algorithm. For ease of exposition, we will again utilize Figure 2 to demonstrate the operation of DRA. Let us focus on the perimeter sensor $s_{1}$ (although a similar discussion applies to the other perimeter nodes as well.) The DRA algorithm starts in the first step by discovering an adequate number of votes (candidate neighbors) for each perimeter sensor $s_{i}$ (lines 2-6). This is done by probing the 1-hop core node neighbors of $s_{1},\left(N H\left(s_{1}\right)\right)$, which are $s_{4}$ and $s_{5}$ (line 3$)$. If the number of neighboring nodes, $\left|N H\left(s_{1}\right)\right|$ is lower than a user-defined threshold vmin (for our discussion let vmin=4) then $s_{1}$ expands its neighbors by incorporating more multi-hop nodes (line 5). That results in the increase of the $N H\left(s_{1}\right)$ set (i.e., $s_{6}$ and $s_{12}$ are added to $N H\left(s_{1}\right)$ ). Besides the identifier of each neighbor, $s_{1}$ also stores the hop count for each of them (i.e., $\left.\left(s_{4}, 1\right),\left(s_{5}, 1\right),\left(s_{6}, 2\right),\left(s_{12}, 2\right)\right)$ so that it can later decide which set of neighbors will produce the most energyefficient replication strategy. Since the number of candidates in $N H\left(s_{1}\right)$ is 4 , thus the vmin requirement has been satisfied, $s_{1}$ utilizes all of these 4 nodes including itself (i.e., $v_{i}=5$ ). Next, $s_{1}$ proceeds with the selection of a subset of $v_{i}$ for data 


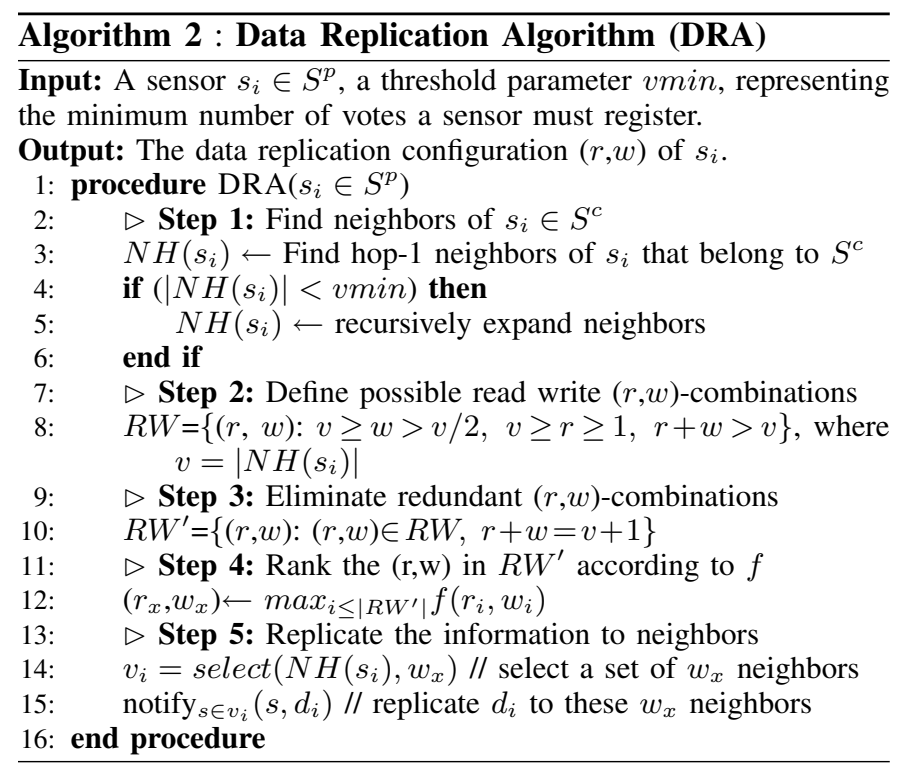

replication. This is done by utilizing a voting process that operates as follows (we denote $\left|v_{i}\right|$ as $v$ for brevity):

In Step 2 we define two integers, $r$ (number of read operations) and $w$ (number of write/replicate operations) with the following properties:

$$
r+w>v, \quad v \geq r \geq 1, \quad v \geq w>v / 2
$$

We then create the $R W$-set of eligible $(r, w)$-combinations (line 8). In our example, since $w$ needs to be in the range $5 \geq$ $w>2.5$ then $w \in\{3,4,5\}$. Furthermore, since $r+w>v$ then $r>v-w$ and consequently the following $(r, w)$-combinations are valid: $R W=\{(1,5),(2,5),(3,5),(4,5),(5,5),(2,4),(3,4)$, $(4,4),(5,4),(3,3),(4,3),(5,3)\}$.

In Step 3 of the voting process, we aim to eliminate redundant $(r, w)$-combinations in the $R W$ set. To understand the intuition behind this elimination consider the $(1,5)$ combination. Since $w=5$ (i.e., all sensors hold a replica of datum $d_{1}$ ) then it is redundant to read more replicas than one (i.e., $(2,5),(3,5), \cdots,(5,5)$ are redundant). Although all of these combinations can recover $d_{i}$ in cases of failures, they do not have the same energy requirements and should thus be excluded from the $R W$ set. For instance the $(2,5)$-combination requires 1 read more than the $(1,5)$-combination and should thus be eliminated. The elimination of redundant combinations yields $R W^{\prime}=\{(1,5),(2,4),(3,3)\}$.

The objective of Step 4 is to further prune the $R W^{\prime}$ set in order to derive the $(r, w)$-combination that requires the least possible energy, but this operation is not straightforward. On the one hand, having more $w$ operations involved in the replication process increases the overall fault-tolerance. On the other hand, more $w$ operations would also incur additional messaging and consequently would require more energy. The negative effect of more $w$ operations is particularly more apparent in cases where nodes have a hop distance from $s_{i}$ that is larger than 1 (i.e., are not 1-hop neighbors).

In the fourth step of the DRA algorithm, we rank the remaining $R W^{\prime}=\{(1,5),(2,4),(3,3)\}$ combinations using a ranking function $f_{(r, w)}$ and choose the one with the highest score. In particular, the local ranking proceeds as follows:

- Calculate the number of broadcast messages $\left(n b m_{(r, w)}\right)$ that would be required for the replication process of the remaining $(r, w)$-combinations $\in R W^{\prime}$ using the hopcount information gathered during lines 2-6 of DRA. Normalize $\mathrm{nbm}_{(r, w)}$ to [0..1] using the following function: $n b m_{(r, w)}^{\prime}=\min \left(n b m_{\forall(r, w)}\right) / n b m_{(r, w)}$.

- Calculate the replication spreading factor $\left(r s f_{(r, w)}\right)$ by normalizing the $w$ of each combination to [0..1] using formula $w / \max \left(\forall w \in R W^{\prime}\right)$.

- Calculate the rank of each $(r, w)$-combination by summing the number of broadcast messages and replication spreading factor parameters: $f_{(r, w)}=n b m_{(r, w)}^{\prime}+$ $r s f_{(r, w)}{ }^{3}$

The results of the ranking on our example are summarized in Table II. The presented results indicate that the $(1,5)$ combination has the highest rank in the $f$ function and consequently that plan is utilized for the replication of $d_{i}$.

TABLE II

Ranking the $(r, w)$-combinations of $R W^{\prime}$ during the fourth step of DRA

\begin{tabular}{|c|r|r|r|r|}
\hline$(r, w)$ & $n b m_{(r, w)}$ & $n m_{(r, w)}^{\prime}$ & $r s f_{(r, w)}$ & $f_{(r, w)}$ \\
\hline \hline$(\mathbf{1 , 5})$ & $\mathbf{4}$ & $\mathbf{1 . 0}$ & $\mathbf{1 . 0}$ & $\mathbf{2 . 0}$ \\
$(2,4)$ & 5 & 0.8 & 0.8 & 1.6 \\
$(3,3)$ & 4 & 1.0 & 0.6 & 1.6 \\
\hline
\end{tabular}

In the final fifth step of DRA, $s_{i}$ proceeds with the replication of $d_{i}$ to the identified neighboring nodes. In particular, in line $14 s_{i}$ selects $w_{x}$ neighbors from its $N H\left(s_{i}\right)$ list and stores these results in the $v_{i}$ set. Each $s_{i}$ then proceeds with the replication of $d_{i}$ to the identified $w_{x}$ nodes in line 15 . This completes the operation of the DRA algorithm.

Theorem 1: The DRA algorithm guarantees that a datum $d_{i}$ can be recovered if the number of reads $\left(r_{x}\right)$ from the votes of $s_{i}$ is at least $v-w_{x}+1\left(v \geq w_{x}\right)$, where $v$ denotes the number of all votes and $w_{x}$ the number of writes during the replication of $d_{i}$.

Proof: Let us select first two sets, $R$ and $W$, such that $|R|=$ $r_{x}$ and $|W|=w_{x}\left(R, W \subset v_{i}\right)$ as dictated by DRA. Since $w_{x}>v / 2$ then $d_{i}$ has been replicated to more than half of the nodes assigned a vote by node $i$. Now, considering that $r_{x}+w_{x}>v$, we must have $R \cap W \neq \emptyset$. Hence any read operation is guaranteed to read the value of at least one copy which has been updated by the latest write $\square$

\section{EXPERIMENTAL EVALUATION}

In this section we present our experimental evaluation of the SenseSwarm framework.

\footnotetext{
${ }^{3} \mathrm{nbm}_{(r, w)}^{\prime}$ and $r s f_{(r, w)}$ are the two most prominent parameters for selecting the best $(r, w)$-combination. However, one could also consider parameters like capacity required to store the datums and recovery performance.
} 


\section{A. Experimental Methodology}

We adopt a trace-driven experimental methodology in which a real dataset from $n$ sensors is fed into our trace-driven simulator. Our methodology is as follows:

Sensing Device: We use the energy model of Crossbow's research sensor device TelosB [5] to validate our ideas. TelosB is a ultra-low power wireless sensor equipped with a $8 \mathrm{MHz}$ MSP430 core, $1 \mathrm{MB}$ of external flash storage, and a $250 \mathrm{Kbps}$ Chipcon (now Texas Instruments) CC2420 RF Transceiver that consumes $23 \mathrm{~mA}$ in receive mode $(\mathrm{Rx}), 19.5 \mathrm{~mA}$ in transmit mode (Tx), $7.8 \mathrm{~mA}$ in active mode (MCU active) with the radio off and $5.1 \mu \mathrm{A}$ in sleep mode. Our performance measure is Energy, in Joules, that is required at each discrete time instance to resolve the query. The energy formula is as following: Energy $($ Joules $)=$ Volts $\times$ Amperes $\times$ Seconds . For instance the energy to transmit 30 bytes at $1.8 \mathrm{~V}$ is: $1.8 \mathrm{~V} \times 23 * 10^{-3} \mathrm{~A} \times 30 * 8$ bits $/ 250 \mathrm{kbps}=39 \mu \mathrm{J}$.

Dataset: We utilize a real dataset from Intel Berkeley Research [13]. This dataset contains data that is collected from 58 sensors deployed at the premises of the Intel Research in Berkeley between February 28th and April 5th, 2004. The motes utilized in the deployment were equipped with weather boards and collected time-stamped topology information along with humidity, temperature, light and voltage values once every 31 seconds. The dataset includes 2.3 million readings collected from these sensors. We use 10,000 readings from the 54 sensors that had the largest amount of local readings since some of them had many missing values.

Swarm Simulation: In order to introduce motion to our sensor network we have derived synthetic spatial coordinates for the $n$ sensors using the Craig Reynolds algorithm [22], which is widely used in the computer graphics community. Using this algorithm we generated 100 individual scenes and during each scene a sensor obtains 100 readings (i.e., $\sigma=\sigma^{\prime}=100$ ). In order to simulate failures we make the assumption that there is a $X \%$ independent probability that a node fails at any given timestamp.

\section{B. Perimeter Cost Evaluation}

In the first experimental series we investigate the efficiency of our distributed PA algorithm compared to the centralized CPA algorithm. Figure 5, presents the aggregate cost (i.e., for the whole network and for all 10,000 timestamps) of the two algorithms for 4 different network sizes 54, 150, 300 and 500. These networks were derived from the initial dataset of 54 nodes using replication of the sensor readings to different initial coordinates. We observe that the PA algorithm consumes in all cases between $85 \%-89 \%$ less energy than the CPA algorithm. This is attributed to the fact that during the computation of $s_{\text {min }}$, the PA algorithm intelligently percolates only one $(x, y)$-pair to the sink rather than all of them. Additionally, we observe that the performance gap between the two algorithms grows substantially with the size of the network. Specifically, for $n=54$ the total energy difference

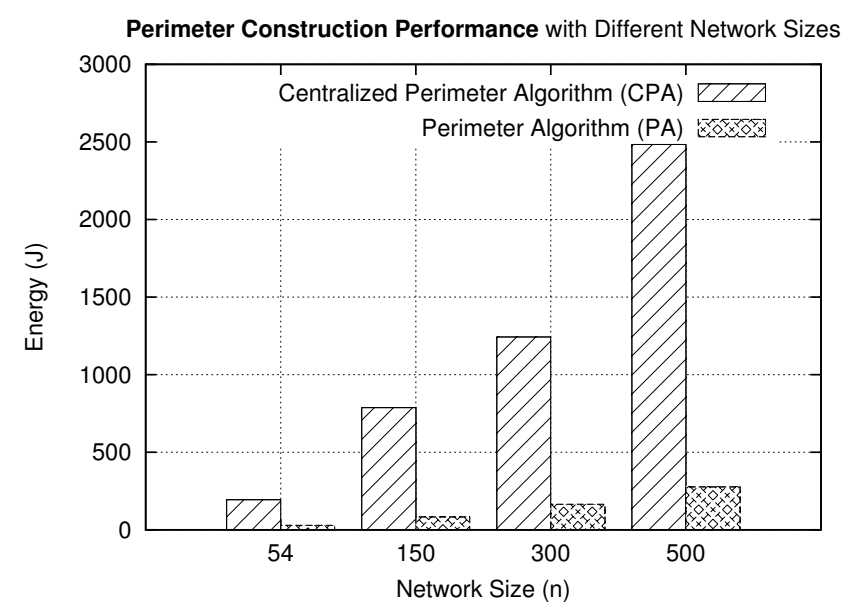

Fig. 5. Evaluating the energy consumption of the Perimeter Algorithm

between the two algorithms was 163 Joules while for $n=500$ the total energy difference was 2,208 Joules.

\section{Replication Phase Evaluation}

In the final experimental series, we evaluate the faulttolerance accuracy of our two replication algorithms.

In the first experiment we measure the absolute faulttolerance accuracy of the Data Replication Algorithm (DRA). To accomplish this, we compare DRA against a version that does not employ any replication strategy, coined NoReplication Algorithm (NRA). We execute both algorithms on each of the individual scenes generated by our swarm simulator. During each one of the 100 individual scenes, we randomly select a sensor node to be the sink. As soon as the sink is selected, it registers 10 random queries each of which requesting events detected by different sets of perimeter sensors. In order to measure the accuracy of each of the algorithms, we measure the average ratio of detected events over the total number of events requested by the 10 queries.

Figure 6, illustrates the fault-tolerance accuracy of the two algorithms over an increasing failure rate. We observe that in all cases DRA maintains a competitive advantage of $\approx 19$ $48 \%$ over NRA. This is due to the voting-based replication strategy utilized by DRA. Note that we have configured DRA with vmin $=3$ (i.e., 3 votes). Since, in DRA, detected events are replicated to 3 neighboring nodes, even if a node fails, its detected events are easily obtained by its votes thus ensuring a higher level of accuracy. We also observe that with a $60 \%$ failure rate the accuracy of both algorithms starts to decrease rapidly. This is expected at such high failure rates as large segments of the query routing tree become inaccessible by the sink.

We have finally measured the number of extra communication messages that DRA requires during replication. We discovered that on average, DRA requires approximately $90 \pm 32$ extra messages (i.e., has a message complexity of $O(n)$ ). 


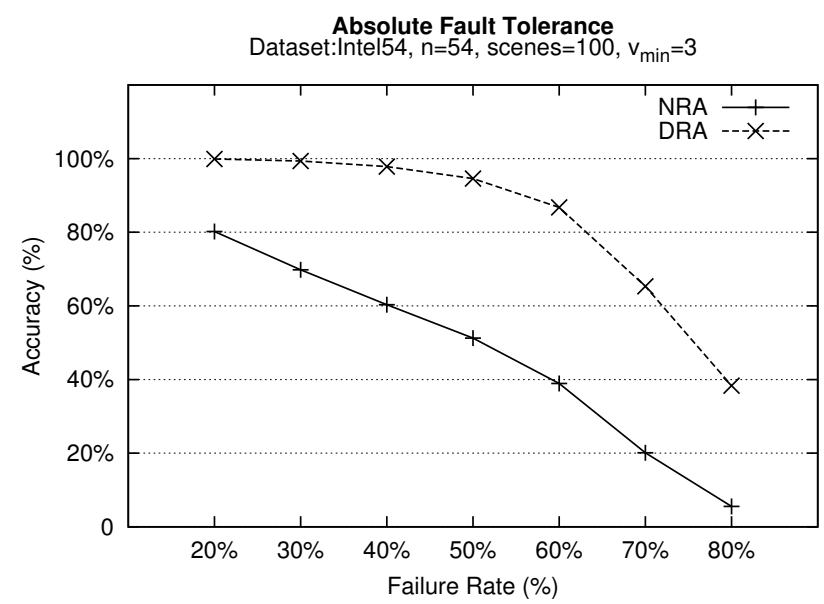

Fig. 6. Evaluating the absolute fault-tolerance accuracy (that measures the percentage of datums that can be recovered) for the DRA and NRA algorithms.

\section{CONCLUSions AND Future WORK}

This paper introduces and formalizes a novel perimeterbased data acquisition framework for mobile sensor networks, coined SenseSwarm. SenseSwarm dynamically partitions the sensing devices into perimeter and core nodes. Data acquisition is scheduled at the perimeter, with the invocation of the PA algorithm, while storage and replication takes place at the core nodes, with the invocation of the DRA algorithm. Our trace-driven experimentation with realistic data shows that our framework offers tremendous energy reductions while maintaining high data availability rates. In particular, we found that even with $60 \%$ system failures we can recover over $80 \%$ of generated events exactly. In the future we plan to study other geometric shapes besides MBRs, different sink selection strategies for in-network replication and also techniques to incrementally maintain the perimeter rather than reconstructing it in every iteration.

Acknowledgements: This work was supported in part by the Open University of Cyprus under the project SenseView, the US National Science Foundation under the project AQSIOS (\#IIS-0534531), the European Union under the project mPower (\#034707), IPAC (\#224395) and CONET (\#224053), and the Cyprus national project GEITONIA (\#П $\Lambda$ ҮН $/ 0506 / 31)$.

\section{REFERENCES}

[1] Aly M., Pruhs K., Chrysanthis P.K., "KDDCS: a load-balanced innetwork data-centric storage scheme for sensor networks", In CIKM, 2006.

[2] Bergbreiter, S.; Pister, K.S.J., "CotsBots: An Off-the-Shelf Platform for Distributed Robotics,", In IROS, 2003.

[3] Chintalapudi K. and Govindan R., "Localized Edge Detection In Sensor Fields", Ad-hoc Networks, 2003.

[4] Cormen T.H., Leiserson C.E., Rivest R.L., and Stein C., "Introduction to Algorithms", $2^{\text {nd }}$ edition. The MIT Press and McGraw-Hill, 2001.

[5] Crossbow Technology Inc. http://www.xbow.com/

[6] Chrysanthis P.K. and Labrinidis A., "NSF Workshop on Data Management for Mobile Sensor Networks Report”, Jan. 16-17, 2007, Pittsburgh, USA, http://www.mobisensors.org
[7] Dantu K., Rahimi M.H., Shah H., Babel S., Dhariwal A., and Sukhatme G.S., "Robomote: Enabling mobility in sensor networks", In IPSNSPOTS, 2005.

[8] Eriksson, J., Girod, L., Hull, B., Newton, R., Madden, S. and Balakrishnan H., "The Pothole Patrol: Using a Mobile Sensor Network for Road Surface Monitoring", In ACM Int. Conf. on Mobile Systems, Applications And Services 2008.

[9] Hasan A., Pisano W., Panichsakul S., Gray P., Huang J-H., Han R., Lawrence D. and Mohseni K., "SensorFlock: An Airborne Wireless Sensor Network of Micro-Air Vehicles", In ACM Sensys, 2007

[10] Hill J., Szewczyk R., Woo A., Hollar S., Culler D., Pister K., "System Architecture Directions for Networked Sensors", In SIGOPS Operating Systems Review, Vol.34, No.5, pp.93-104, 2000.

[11] Hull B., Bychkovsky V., Chen K., Goraczko M., Miu A., Shih E., Zhang Y., Balakrishnan H., and Madden S., "CarTel: A Distributed Mobile Sensor Computing System", In ACM Int. Conf. on Embedded Networked Sensor Systems, 2006.

[12] Intanagonwiwat C., Govindan R. Estrin D., "Directed diffusion: A scalable and robust communication paradigm for sensor networks", In ACM MobiCom, 2000.

[13] Intel Lab Data http://db.csail.mit.edu/labdata/labdata.html

[14] Jalodia S., Mutchler D., "Dynamic Voting Algorithms for Maintaining the Consistency of a Replicated Database", IN ACM TODS, vol.15, pp.230-280, June, 1990.

[15] Koren I., Krishna C.M., "Fault-Tolerant Systems", Elsevier, 2007.

[16] Navarro-Serment, L.E., Grabowski, R., Paredis, C.J.J., and Khosla, P.K. "Millibots: The Development of a Framework and Algorithms for a Distributed Heterogeneous Robot Team,", IEEE Robotics and Automation Magazine, Vol. 9, No. 4, December 2002.

[17] Nittel S., Trigoni N., Ferentinos K., Neville F., Nural A., Pettigrew $\mathrm{N}$., "A drift-tolerant model for data management in ocean sensor networks", In ACM MobiDE, 2007.

[18] Madden S.R., Franklin M.J., Hellerstein J.M., Hong W., "The Design of an Acquisitional Query Processor for Sensor Networks", In ACM SIGMOD, 2003.

[19] Madden S.R., Franklin M.J., Hellerstein J.M., Hong W., "TAG: a Tiny AGgregation Service for Ad-Hoc Sensor Networks", In Usenix OSDI, Vol.36, pp.131-146, 2002.

[20] Mani A., Rajashekhar M., Levis P. "TINX: a tiny index design for flash memory on wireless sensor devices", In ACM Sensys, 2006

[21] Srinivasan S., Ramamritham K., Kulkarni P., "ACE in the Hole: Adaptive Contour Estimation Using Collaborating Mobile Sensors", In IEEE IPSN, 2008.

[22] Reynolds, C. W., "Flocks, Herds, and Schools: A Distributed Behavioral Model", In ACM SIGGRAPH, 1987.

[23] Sadler C., Zhang P., Martonosi M., Lyon S., "Hardware Design Experiences in ZebraNet", In ACM SenSys, 2004.

[24] Shenker S., Ratnasamy S., Karp B., Govindan R., Estrin D., "Datacentric storage in sensornets", In SIGCOMM Computer Communication Review, Vol. 33 , Iss. 1, pp.137-142, 2003.

[25] Szewczyk R., Mainwaring A., Polastre J., Anderson J., Culler D., "An Analysis of a Large Scale Habitat Monitoring Application", In ACM SenSys, 2004.

[26] Yao Y., Gehrke J.E., "The cougar approach to in-network query processing in sensor networks", In SIGMOD Record, Vol.32, No.3, pp.9-18, 2002.

[27] Zeinalipour-Yazti D., Andreou P., Chrysanthis P. and Samaras G., "MINT Views: Materialized In-Network Top-k Views in Sensor Networks", In IEEE MDM, 2007.

[28] Zeinalipour-Yazti D., Andreou P., Chrysanthis P.K., Samaras G., "SenseSwarm: a perimeter-based data acquisition framework for mobile sensor networks", In VLDB's DMSN, 2007.

[29] Zeinalipour-Yazti D., Chrysanthis P.K., "Mobile Sensor Network Data Management" Book Chapter in the Encyclopedia of Database Systems (EDBS), Ozsu, M. Tamer; Liu, Ling (Eds.), ISBN: 978-0-387-49616-0, 2009.

[30] Zeinalipour-Yazti D., Lin S., Kalogeraki V., Gunopulos D., Najjar W., "MicroHash: An Efficient Index Structure for Flash-Based Sensor Devices", In USENIX FAST, 2005. 